O26:H11 (rfbO26, fliC11); E. coli O111 - to 2 serovars: O111:H8 (5 strains $r f b \mathrm{O} 111, f l i C 8)$ and O111:H2 (12 strains of $r f b \mathrm{O} 111, f l i C 2)$. E. coli $\mathrm{O} 55$ also belonged to 2 serovars: O55:H7 (one strain, $r f b \mathrm{O} 55, \mathrm{fliC} 7$ ) and O55:H6 (five strains of $r f b \mathrm{O} 55$, fliC6). According to our data, 10 strains of $\mathrm{O} 26: \mathrm{H} 11,5$ strains of $\mathrm{O} 111: \mathrm{H} 8$ and 1 strain of $\mathrm{O} 55: \mathrm{H} 7$ had stx 1 gene (encoding the production of shiga-like toxin 1) in combination with eae gene (the adhesion factor, intimin) and could be consider as EHEC. All strains E. coli $\mathrm{O} 111: \mathrm{H} 2$ and $E$. coli $\mathrm{O} 55: \mathrm{H} 6$ and 42 E. coli $\mathrm{O} 26$ : H11 had only eae gene, indicating that these strains belonged to the EPEC.

The introduction of molecular methods of serotyping and detection of virulence factors in laboratory diagnostics makes it possible to confirm the true pathogenicity of $E$. coli strains and to minimize the diagnostic errors in etiological interpretation of acute enteric infections.

\section{COMPARISON OF PHENOTYPIC} AND MOLECULAR-GENETIC PROPERTIES OF THE STRAINS NEISSERIA MENINGITIDIS ISOLATED FROM PATIENTS WITH GENERALIZED FORMS OF MENINGOCOCCAL INFECTION AND CARRIERS

\section{E.A. Martens, S.V. Sidorenko, L.I. Zhelezova}

Pediatric Research and Clinical Center for Infectious Diseases, St. Petersburg, Russia

Characterization of isolates of Neisseria meningitidis obtained from patients with meningococcal disease or from nasopharyngeal swabs of asymptomatic carriers can be achieved by several methods which provide different levels of discrimination.

A total of 42 gram-negative, oxidase-positive diplococcus strains isolated from individuals with meningococcal disease in 2009-2018 years and 65 isolates from 1075 nasopharyngeal carriers in 2016-2018 years were examined by three approaches: serological typing by agglutination, determination of the serogroups by real-time PCR, multilocus sequence typing (MLST). Each strain from patients with meningococcal disease was also determined sensitivity to antibiotics by dilution in broth.

The majority of strains isolated from patients belonged according to the results of agglutination and real-time PCR data to serogroup B (50 and $40.5 \%$ respectively), $\mathrm{C}$ (16.7 and $11.9 \%$ respectively) and $\mathrm{W}$ (14.3\% by results of both methods). Among the isolates from carriers according to the results of agglutination and real-time PCR data were dominated serogroup $\mathrm{W}$ (37.0 and $30.8 \%$ respectively) and B (32.3 and $26.2 \%$ respectively). Invasive isolates of serogroup B were resistant to penicillin (28.6\%), levofloxacin (33.3\%), chloramphenicol $(27.3 \%)$, rifampicin (14.3\%), invasive isolates of serogroup W135 to chloramphenicol $(9.1 \%)$.

MLST established the genetic relationships of the isolates from patients and identified members of known hypervirulent lineage CC11 $(n=4)$.

Six isolates of $N$. meningitidis (invasive and from nasopharyngeal swab) were additionally investigated by whole genome sequencing.

The results are included in the GenBank international database: SRR7352647 SAMN09435696 Nm-146 blood 16-Apr-2018, SRR7352646 SAMN09435695 Nm-105 nasopharyngeal swab Aug-2016 (https://www.ncbi.nlm.nih. gov/sra/SRP150714)

\section{MOLECULAR TYPING IN RESEARCH OF EPIDEMICAL} CHOLERA MANIFESTATION

L.V. Mironova, Zh.Yu. Khunkheeva, A.S. Ponomareva, A.S. Gladkikh, N.O. Bochalgin, E.A. Basov, S.V. Balakhonov Irkutsk Antiplague Research Institute of Rospotrebnadzor, Irkutsk, Russia

The causative agent of El Tor cholera evolved adaptive mechanisms to ensure its' preservation and accumulation in certain ecological niches and provide existence of its population in various climatic and geographical zones. Considering this, epidemics development mechanism differs in endemic and non-endemic areas. Siberian and the Far East regions are non-endemic territories for cholera. The last epidemic complications in this region were reported in 1990s and had a form of certain infection importation cases and acute outbreaks, associated with importations. Along with this, strains of the Vibrio cholerae El Tor, devoid of the pathogenicity determinants, are found annually in environmental objects.

The aim of this work is to elucidate the patterns of cholera epidemiological manifestations in Siberia and the Far East, based on a complex molecular genetic analysis of $V$. cholerae El Tor strains.

In complex assay, using amplification profiling, MLVA-, PFGE-, MLST-, and wgSNP-typing, we found, that $V$. cholerae El Tor strains isolated in epidemic complications, homogenous in basic pathogenicity, pandemicity, persistence determinants along with nucleotide context of housekeeping genes, are characterized by diversity in the associated with pathogenicity genomic loci structure, macrorestriction patterns, SNP-profiles, and structure of variable tandem repeats loci. At the same time, closely related subclones of one clonal variant were identified within the individual outbreaks. Considering the typing data, we can conclude that the outbreaks genesis in the non-endemic territories of the region is determined by the hyperinfectious clone importation. Circulation of closely related subclonal vibrio variants during the outbreak can be caused by environmental factors during the implementation of the water or the contact-household transmission routes.

$V$. cholerae $\mathrm{O} 1 \mathrm{El}$ Tor isolates from surface watercourse in a cholera free period are characterized by a significant polymorphism of MLVA profiles and PFGE genotypes; that indicates a high heterogeneity of the water populations of the microorganism and the probability of microevolutionary changes during persistence in surface watercourse, as well as the possible periodic introduction of new clones into aquatic ecosystems.

Thus, molecular approaches in the analysis of cholera manifestations provides an understanding of the epidemic complications development patterns in the territory and $V$. cholerae persistence in the environment.

\section{PCR FOR DIAGNOSIS OF GONOCOCCAL INFECTION: PANACEA OR ESCAPE FROM REALITY}

N.V. Nikolaeva, A.P. Godovalov, T.I. Karpunina

Acad. E.A. Wagner Perm State Medical University, Perm, Russia

The aim of this study was to provide an analytical assessment of the role and place of PCR in gonococcal infection (GI) monitoring. Clinical guidelines define a set of laboratory tests for its diagnosis, including molecular genetic techniques (MGT). It is considered, that MGT have the highest diagnostic sensitivity, in contrast to the traditional procedures (microscopic, bacteriolo- 
gical, immunological). Moreover, the latter do not allow to establish the disease etiology in $20-40 \%$ of cases due to the variability of morphological, cultural properties and antigenic structure of the pathogen [Gushchin A.E. et al., 2014]. The use of MGT had to improve the laboratory diagnostics quality and, as a consequence, to provide a high level of GI detection. According to official statistics, in the Perm region there is a stable reduction of GI incidences. Meanwhile, PCR is used no more than in 25\% cases of GI and is carried out exclusively by commercial laboratories. The PCR modifications used in such institutions do not exclude the appearance of both false-negative and false-positive results due to a number of objective and subjective reasons. Thus, a high degree of nucleotide sequence homology and frequent genetic exchange between gonococci and other species of the genus Neisseria, as well as a high level of genetic polymorphism of different strains of Neisseria gonorrhoeae are the serious problem of molecular diagnosis of GI [Palmer H.M. et al., 2003; Whiley D.M. et al., 2006; Tabrizi S.N. et al., 2011]. The cross-reaction with other microorganisms leads to a false positive results. MGT, which does not involve analysis of strain characteristics (presence of "pathogenicity islands", genetic markers of resistance, etc.), does not provide qualitative monitoring of $N$. gonorrhoeae. In addition, the detectable fragments of nucleic acids do not always belong to living pathogens. Thus, microbiological diagnostics of GI, including the use of PCR, remains an unsolved problem. The needs of practical health care require the improvement of existing approaches and the development of new ones.

This work was supported by RFBR grant No. 17-44-590404 and Administration of Perm Region.

\subsection{4 doi: 10.15789/2220-7619-2018-4-2.14 \\ EXPERIENCE OF EXPANDED PRIMER SET USING FOR DETECTION OF THE PATHOGENIC POTENTIAL OF ESCHERICHIA COLI}

E.A. Orishak ${ }^{1}$, K.G. Kosyakova ${ }^{1}$, L.Ju. Nilova ${ }^{1}$ O.A. Kameneva ${ }^{2}$, A.P. Listopadova ${ }^{3}$, V.P. Novikova ${ }^{3}$, E.G. Oganesyan ${ }^{1}$

${ }^{1}$ North-Western State Medical University named after I.I. Mechnikov, St. Petersburg, Russia; ${ }^{2}$ St. Petersburg Children's City Hospital No. 22, St. Petersburg, Russia $;{ }^{3}$ St. Petersburg State Pediatric Medical University, St. Petersburg, Russia

The purpose of the study to reveal the pathogenic potential of E.coli in routine intestinal dysbiosis diagnostics in children are under regular medical check-up because of chronic conditions by detection of genes encoding pathogenicity factors by polymerase chain reaction (PCR).

9-17 years old children were examined with standard procedure of bacteriological diagnostics of intestinal dysbiosis and subdivided into groups: 1) with chronic gastroduodenitis - 22 patients; 2 ) with atopic dermatitis (AtD) - 19; 3) with bronchial asthma - 7. E.coli DNA was recovered with assay reagent set "Ribo-prep" (Russia). Specific gene sequences of ETEC, EPEC, EHEC, EAEC and $E I E C$ were found by real-time PCR (CFX 96, BioRad, USA) with assay reagent set "Amplisens-Escherichioses" (Russia). Also, individual primers (BioBeagle, Russia) were used to identify genes of pathogenicity factors of $E$. coli, specifically eaeA, $b f p A-$ of EPEC, st1, lt - of ETEC, cnf1 - NMEC, afa - DAEC, ipaH, ial - EIEC, aggA, east1 - EAEC, stxl, stx $2-E H E C$, chuA - of AIEC (sotermed Adherent-Invasive E. coli, associated with Crohn's disease) correspondingly.
In the group of chronic gastroduodenitis E. coli stains with pathogenicity coding genes were detected in 7 of 22 tested strains (genes eaeA, $b f p A, \operatorname{agg} A, a f a, c h u A$ ), in the group with AtD - 3 of 19 (genes eae $A, b f p A, a g g A$, east). Among the children with bronchial asthma, pathogenic Escherichia was not found. In routine intestinal dysbiosis diagnostics virulence coding genes of diarrheagenic $E$. coli were detected in 11 cases (23.9\%).

In order to reveal the etiological significance of Escherichia in children with chronic diseases, as well as to determine their probabilistic role in the manifestation of diseases, the infectious component of which remains poorly studied, the multiplex real-time PCR should be introduce in the laboratory procedure specifications at very least, as well as the creation of primer set based on updated data on diarrheagenic E. coli or sighting of genes, as in the case of Crohn's disease.

2.15 doi: $10.15789 / 2220-7619-2018-4-2.15$

\section{HEPATITIS B VIRUS IDENTIFICATION} IN THE ENSURING INFECTIOUS SAFETY OF BLOOD TRANSFUSIONS

Yu.V. Ostankova, E.B. Zueva, D.E. Valutite

St. Petersburg Pasteur Institute, St. Petersburg, Russia

Ensuring the infectious safety of blood transfusions during planned and urgent surgical operations is a topical medical problem and should be implemented first of all in order to prevent the transmission of viruses. Hepatitis B virus (HBV) is one of the most common hepatotropic viruses that can cause both acute and chronic course of the disease. One form of chronic viral hepatitis B is occult hepatitis $\mathrm{B}$, characterized by the presence of HBV DNA in the liver and undetectable levels of HBsAg and HBV DNA in the peripheral blood. Then in most cases, virus replication and gene expression can be suppressed so much that the viral load in the peripheral blood of the patient is extremely low, up to the impossibility of detecting HBV DNA by standard methods, but no elimination of the virus.

The aim of our study was the identification and genotyping of HBV in blood donors.

The material was blood plasma of 1003 blood donors from two transfusion centers. A method for detecting HBV DNA with a low viral load based on a two-step PCR, followed by sequencing was used.

HBV was detected in $6.14 \%$ of donors. In the region with a high prevalence of $\mathrm{HBV}$, the incidence of occult $\mathrm{HBV}$ in blood donors was $9.4 \%$. In the region with a relatively lower prevalence of $\mathrm{HBV}$, the incidence of occult $\mathrm{HB}$ in blood donors was $4.23 \%$. In phylogenetic analysis among the HBV samples obtained from HBsAg-negative blood donors in a region with a high prevalence of HBV, the following subgenotypes are represented: D1 $-46.8 \%$, D2 - $17.05 \%$, D3 - 31.9\%, A2 - 4.25\%, respectively. In the region with a relatively lower prevalence of $\mathrm{HBV}$ subgenotypes are presented: D $1-22.73 \%$, D3 - 72.73\%, $\mathrm{C}-4.54 \%$. In the case of donors with detected HBV DNA, $\mathrm{HBcor}$ IgG antibodies were detected in $34.7 \%$ of cases. At the same time, in the analysis for serological markers of the whole group, HBcor IgG antibodies were detected in $21.2 \%$ of cases, of which HBV DNA was detected only in $11.3 \%$, which is in agreement with the data on the hyperdiagnosticity of this marker.

The use of molecular diagnostic methods in the blood safety algorithm to detect HBV with low viral load can ensure the viral safety of blood transfusions, especially where viral hepatitis is widespread. 